\title{
Joint Associations of Activity Energy Expenditure and Sedentary Behaviors with Adolescent's Obesity and Dietary Habits
}

By:Al-Hazzaa, HM (Al-Hazzaa, Hazzaa M.) ${ }^{[1]}$; Al-Sobayel, HI (Al-Sobayel, Hana I.) ${ }^{[1]}$;

Abahussain, NA (Abahussain, Nada A.) ${ }^{[\underline{2}]}$; Qahwaji, DM (Qahwaji, Dina M.) ${ }^{[\underline{3}]}$; Al-Haifi, AR (AlHaifi, Ahmad R.) $)^{[4]}$; Desoki, TE (Desoki, Tamer E. $)^{[\underline{5}]}$; Kilani, HA (Kilani, Hashem A.) ${ }^{[\underline{6}]}$; $\underline{\text { Musaiger, AO }}$ (Musaiger, Abdulrahman O. $)^{[\underline{7}]} ; \underline{\text { Kerkadi, A }}$ (Kerkadi, Abdelhamid) ${ }^{[\underline{8}]}$

\section{MEDICINE AND SCIENCE IN SPORTS AND EXERCISE}

Volume: $46 \quad$ Issue: $5 \quad$ Pages: 518-518 Supplement: 1

Meeting Abstract: $1908 \quad$ Published: MAY 2014

Conference: 61st Annual Meeting of the American-College-of-Sports-Medicine

Date: May 027- June 1, 2014

Sponsor(s): Amer Coll Sports Med

Author Information

Addresses:

[ 1 ] King Saud Univ, Riyadh, Saudi Arabia

[ 2 ] Minist Educ, Al Khobar, Saudi Arabia

[ 3 ] King Abdulaziz Univ, Jeddah 21413, Saudi Arabia

[ 4 ] Coll Hlth Sci, Kuwait, Kuwait

[ 5 ] Minist Educ, Dubai, U Arab Emirates

[ 6 ] Sultan Qaboos Univ, Muscat, Oman

[ 7 ] Arab Ctr Nutr, Manama, Bahrain

[ 8 ] Qatar Univ, Doha, Qatar

Publisher

LIPPINCOTT WILLIAMS \& WILKINS, 530 WALNUT ST, PHILADELPHIA, PA 19106-3621 USA

Categories / Classification

Research Areas:Sport Sciences

Web of Science Categories:Sport Sciences

Document Information

Document Type:Meeting Abstract

Language:English

Accession Number: WOS:000339115903267

ISSN: 0195-9131

eISSN: 1530-0315 


\section{Joint Associations of Activity Energy Expenditure and Sedentary Behaviors with Adolescent's Obesity and Dietary Habits.}

Hazzaa M. Al-Hazzaa, FACSM ${ }^{1}$, Hana I. Al-Sobayel ${ }^{1}$, Nada A. Abahussain², Dina M. Qahwaji ${ }^{3}$, Ahmad R. Al-Haifi ${ }^{4}$, Tamer E. Desoki ${ }^{5}$, Hashem A. Kilani ${ }^{6}$, Abdulrahman O. Musaiger ${ }^{7}$, Abdelhamid Kerkadi $^{8}$.

${ }^{1}$ King Saud University, Riyadh, Saudi Arabia. ${ }^{2}$ Ministry of Education, Al-Khobar, Saudi Arabia. ${ }^{3}$ King Abdulaziz University, Jeddah, Saudi Arabia. ${ }^{4}$ College of Health Sciences, Kuwait, Kuwait. ${ }^{5}$ Ministry of Education, Dubai, United Arab Emirates. ${ }^{6}$ Sultan Qaboos University, Muscat, Oman. ${ }^{7}$ Arab Center for Nutrition, Manama, Bahrain. ${ }^{8}$ Qatar University, Doha, Qatar. (No relationships reported)

PURPOSE: Physical inactivity and sedentary behaviors are thought to be independent entities and differently associate with adverse health outcomes. Thus, the aim of this study was to investigate the joint associations of physical activity and sedentary behaviors with obesity indices and dietary habits among adolescents from the Gulf Cooperation Council (GCC) countries.

METHODS: Data were from the Arab Teens Lifestyle Study (ATLS), a school based, cross-sectional lifestyle study. The present analysis included 6279 adolescents (49.4\% males) aged 14-20 years, randomly selected from eight major cities in the GCC countries, using a multistage stratified cluster sampling technique. Anthropometric and self-reported lifestyle data were obtained from participants. Adolescents were classified into four categories: high active \& low sedentary (HA-LS), high active \& high sedentary (HA- HS), low active \& low sedentary (LA-LS) and low active \& high sedentary (LA-HS), based on cut off scores of total activity energy expenditure and daily screen time above and below 1680 METs-min/week and above or below 3 hours/day, respectively.

RESULTS: Results of MANCOVA tests controlling for age revealed that compared with those with LA-HS, adolescents with HA-LS had signifi cantly (<0.001) lower mean (SD) values for BMI (22.6 (5.5) vs $23.7(6.2))$, waist to height ratio $(0.45(.07)$ vs $0.48(.08))$, and less frequent intakes of sugarsweetened drinks (3.7 (2.5) vs 4.5 (2.3)), fast foods (2.2 (1.9) vs $2.9(1.9))$, French fries/potato chips (2.1 (2.0) vs $2.9(2.1))$, cakes/donuts (2.4 (2.1) vs $2.7(2.1))$ and sweets (2.5 (2.1)) vs 3.8 (2.3) but more frequent intakes of breakfast $(3.9(2.7)$ vs $3.3(2.6))$, vegetables $(4.4(2.3)$ vs $3.5(2.4))$, fruits (4.2 (2.3) vs $2.8(2.2))$ and milk (4.3 (2.5) vs $3.6(2.5))$.

CONCLUSIONS: Adolescents with combined high activity energy expenditure and low sedentary behaviors tend to have lower risk of obesity and have more favorable (healthy) dietary habits. These findings carry important implications for adolescent's health promotion and obesity prevention. 